\title{
Editorial
}

\section{High Energy Physics in Underground Labs}

\author{
Bogdan Mitrica, ${ }^{1}$ Maury Goodman, ${ }^{2}$ and Jacek Szabelski ${ }^{3}$ \\ ${ }^{1}$ Horia Hulubei National Institute for Physics and Nuclear Engineering, 077125 Magurele, Romania \\ ${ }^{2}$ Argonne National Laboratory, 9700 South Cass Avenue, Argonne, IL 60439-4803, USA \\ ${ }^{3}$ National Centre for Nuclear Research, Ulica Uniwersytecka 5, 90-137 Lodz, Poland
}

Correspondence should be addressed to Bogdan Mitrica; mitrica@nipne.ro

Received 9 May 2013; Accepted 9 May 2013

Copyright (C) 2013 Bogdan Mitrica et al. This is an open access article distributed under the Creative Commons Attribution License, which permits unrestricted use, distribution, and reproduction in any medium, provided the original work is properly cited.

The field of high energy physics covers a large area of modern research, such as astrophysics, cosmic rays, neutrino oscillations, dark matter, and cosmology. In order to suppress backgrounds, many modern experiments are located in underground sites around the world: Fermilab (USA), Kamioka (Japan), Gran Sasso (Italy), and others. Some modern experiments are now running: Super-Kamiokande, T2 K (Japan), MINOS (USA), and so forth. Others are in development: LBNE, LAGUNA, Hyper-Kamiokande, INO, and so forth.

This special issue brings together information and new data from the modern underground experiments all over the world and covers a large number of related subjects such as dark energy, neutrino oscillations, underground research facilities, very large underground detectors, and modern techniques in particle detection. This special issue covers issues related to both theoretical and experimental aspects of these problems.

In the paper "Charge coupled devices as particle detectors," D. A. Iordache et al. present a theoretical contribution about charge coupled devices which can be used as particle detectors. A new and improved dark current spectroscopy (DCS) method brings us new information regarding the semiconductor particle detectors.

In the paper "Precise $3 D$ track reconstruction algorithm for the ICARUS T600 liquid argon time projection chamber detector," M. Antonello et al. present the status and quality of the Lar TPC detectors during the ICARUS T600 operation. Proposed by C. Rubbia, the Lar TPC detector idea brings a new method for spatial and calorimetric measurement of charged particle interactions.
The paper entitled "The structure of an automatic decision system for a large number of independent particle detectors" by A. R. Sterian presents a statistical model for signal processing and sampling in the case of a large number of independent particle detectors (LNIPDs). An automatic decision system has been analysed, and it is estimated that it could be used for a large number of modern experiments.

In the paper "Site assessment for astroparticle detector location in evaporites of the Polkowice-Sieroszowice copper ore mine, Poland," J. Slizowski et al. present an evaluation of the possibility to excavate a large underground camera for the GLACIER detector as part of the LAGUNA study. The authors show that the Polkowice-Sieroszowice copper ore mine, Poland, is a realistic location for a GLACIER type detector.

In the article "Classic (nonquantic) algorithm for observations and measurements based on statistical strategies of particles fields" by D. Savastru et al., some algorithms for selection and estimation based on statistical hypothesis have been investigated. A new algorithm is presented in detail and can be used for astroparticle detection.

The paper "Realistic approach of the relations of uncertainty of heisenberg," by P. E. Sterian treats an important theoretical problem regarding the Heisenberg's uncertainty in the view of modern experiments. The article brings an original and new idea about the quantum problem of simultaneous measurements of position and momentum.

In the research article "Simulations of muon flux in Slanic salt mine" by M. Bektasoglu et al., new Monte Carlo simulation results regarding the directional muon flux in the underground Slanic Prahova salt mine are presented. 
The simulations are based on GEANT 4 code and take into consideration a realistic topography of the mine. The resulting code is an important tool for anyone interested in the area of underground muon flux research.

The article entitled "Status and new data of the geochemical determination of the pp-neutrino flux by LOREX" by M. K. Pavićević et al. shows the state of the art for LORandite EXperiment (LOREX). The paper presents the latest results and the future plans for this experiment.

The paper "Exclusive reconstruction of $\beta$-decays with missing neutrals" by M. Dima treats a modern and important problem of $\beta$-decays based on a new way to investigate momentum conservation. The method can be used also for other particle physics experimens.

The article "Perspectives on entangled nuclear particle pairs generation and manipulation in quantum communication and cryptography systems" by $\mathrm{O}$. Dănilă et al. is focused on the problem of the two quantum elements phenomenon which can be used for quantum cryptography and physical characterization of the universe.

In the research article "A mobile detector for muon measurements based on two different techniques" by B. Mitrica et al., measurements of the underground muon flux performed with a mobile detector are presented. Two possible configurations using wavelength shifters and PMTs and optical fibers which are then read out by a single PMT are presented. Results obtained with both configurations were compared.

The paper "Cosmic muon detection for geophysical applications" by L. Oláh et al. presents a possible direct application of muon flux measurements focused issues related to the geophysical investigation of mountains.

The papers included in this special issue cover a small number of the diverse issues faced by underground experiments. The theoretical and experimental issues covered here will impact the future of particle physics and lead to further studies that help us understand the constituents of the universe.

Bogdan Mitrica

Maury Goodman Jacek Szabelski 

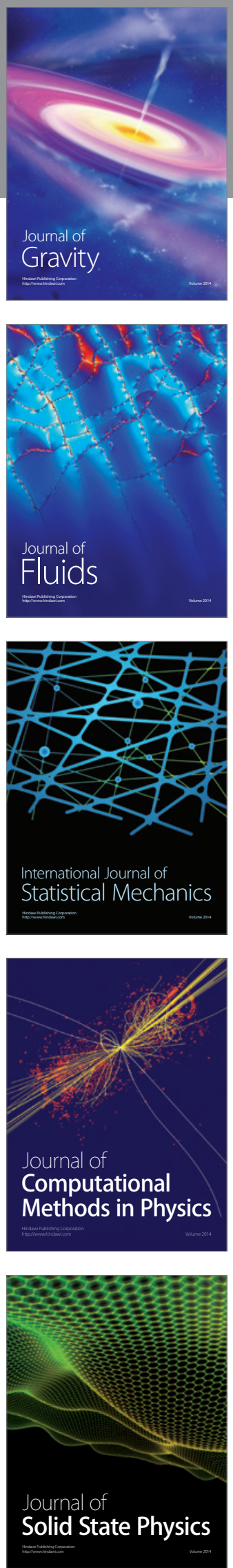

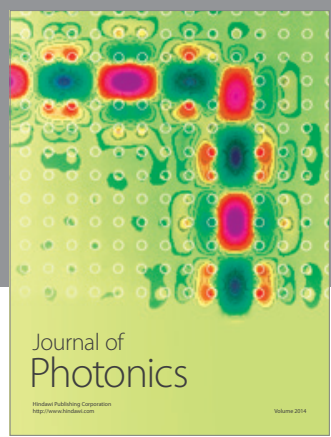

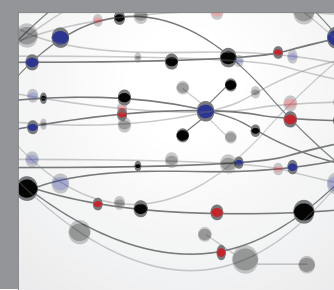

The Scientific World Journal

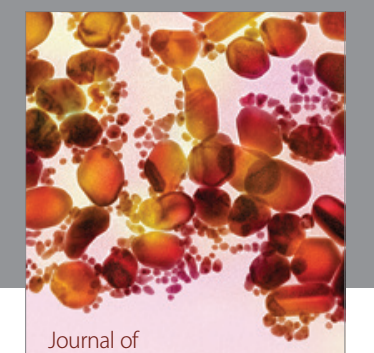

Soft Matter
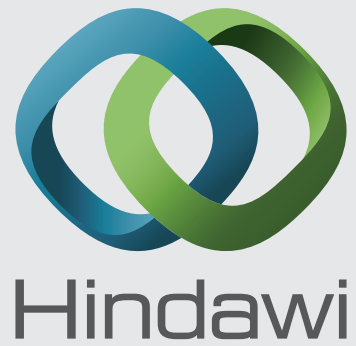

Submit your manuscripts at

http://www.hindawi.com
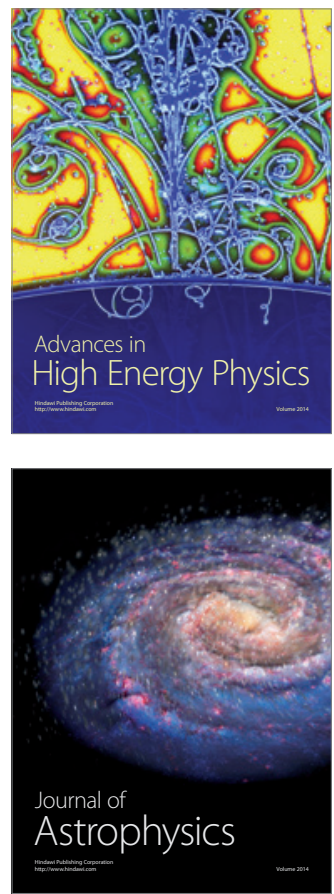


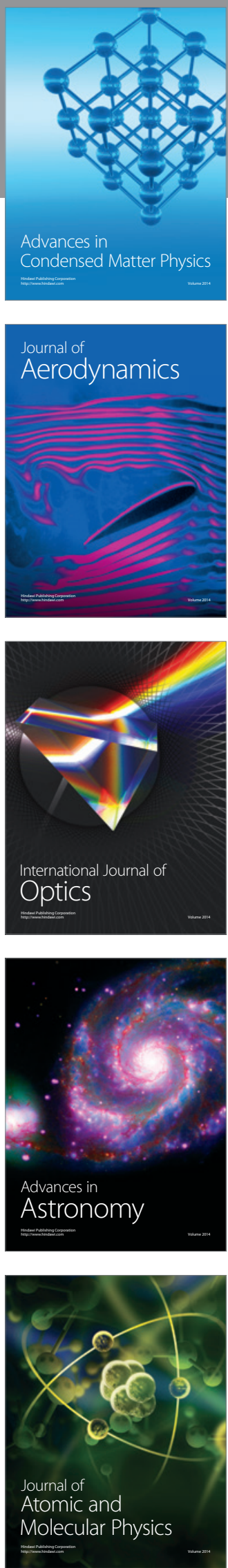